\title{
Using Mapping, Global Positioning System (GPS) and Smart Phone Technology in Rabies Control Program in Kotagiri, Nilgiris District, India
}

\author{
E. Pandey ${ }^{1, *}$, D.K. Singh ${ }^{2}$, R. Raut ${ }^{3}$ \\ ${ }^{1}$ Vet for Your Pet Small Animal Clinic, Kathmandu, Nepal \\ ${ }^{2}$ Institute of Agriculture and Animal Science, Tribhuvan University, Nepal \\ ${ }^{3}$ Agriculture and Forestry University, Rampur, Chitwan, Nepal \\ *Corresponding author: ekatapandey75@gmail.com
}

\begin{abstract}
In this study, we utilized a catch-vaccinate-release approach for dogs in a canine rabies vaccination program in Kotagiri municipality, India. Following vaccination, surveys on dog population and their vaccination status was undertaken. A bespoke smartphone 'Mission Rabies' application was developed to facilitate data entry and team management. This global positioning system (GPS) enabled application captured the location of all vaccinated dogs and dogs sighted on post-vaccination surveys. In areas where coverage was below $70 \%$, catching teams were re-deployed to vaccinate additional dogs followed by repeat survey. Out of 248 dogs captured, only 210 dogs were vaccinated because of the previous vaccination history within a year, and refusal of owner due to their misconceptions. In survey, 147 dogs were sighted of which 77 were seen marked with paints indicating a vaccination coverage of $52.4 \%$. The total estimated dog population was found to be 475. Our study demonstrated that mobile technology enabled efficient team management and real-time data entry and analysis. The vaccination approach outlined in this study can serve as a guideline for rapid vaccination of large numbers of dogs with a high coverage rate in free roaming dog populations in India in the future.
\end{abstract}

Keywords: Rabies, Vaccination, Mark, Capture, Dog, Population

\section{INTRODUCTION}

Rabies is one of the deadliest zoonotic diseases responsible for more than 59,000 human deaths annually across the world (Fooks et al., 2014, Hampson et al., 2015). It is also the most lethal infectious disease with a case fatality rate of $100 \%$ even with advanced medical intervention (Fooks et al., 2014). Rabies is a viral neurological disease affecting all mammals, including human beings. Canine rabies has been reported as one of the neglected diseases in the developing world (Lankester F et 
al., 2014). The annual global cost of rabies has been estimated to be 8.6 billion US dollars and accounts for over 3.7 million disability-adjusted life years (Hampson $\mathrm{K}$ et al., 2015).

In many developing countries, dogs are allowed to roam freely and are the principal reservoir for the disease, with almost all human cases of rabies contracted from bites of an infected dog (WHO, 2013). However, this disease is preventable. Mass vaccination of the dog population has been shown to be effective in eliminating this disease from many countries (Hampson et al., 2015, Vigilato et al., 2013 and Wells, 1954). This has led to the broad belief that the global elimination of canine transmitted rabies is possible through mass dog vaccination (Hampson et al., 2009, Lembo et al., 2010 and Morters et al., 2014). Despite the feasibility of eliminating both canine and human rabies through widespread canine vaccination programmes, there is still limited investment for large scale dog vaccinations in endemic areas of many African and Asian countries (Hampson et al., 2015).

India alone accounts for over $35 \%$ of the global rabies burden with over 20,000 annual deaths. Despite the need to develop and undertake mass canine vaccination programmes in India, there are few published reports of successful implementation of large-scale vaccination programmes (Kakkar et al., 2012, Shahid et al., 2015). There is a dearth of research relating to the Indian free-roaming dog population and practical implementation of effective mass canine vaccination on a scale that could be broadened to a state-wide or even national level (Belsare et al., 2013, Davlin et al., 2013 and Abbas et al., 2014). A major challenge to the eradication of rabies in India is ensuring epidemiologically required vaccination coverage to break the Rabies transmission cycle in the dog population. There is a broad consensus that over $70 \%$ vaccination coverage in an area is sufficient to break the transmission cycle and reduce the incidence of rabies in dog and human populations (WHO, 2013).

Central point vaccination campaigns have been effective at accessing a large enough proportion of the dog population and make an impact on canine and human rabies incidence in parts of Africa (Jibat et al., 2015). However, these approaches have been ineffective in reaching a high proportion in Indian dog population where the majority of dogs are free roaming (Belsare et al., 2013). To progress towards the eradication of canine transmitted rabies in India, it is essential that effective field protocols are developed which facilitate mass canine vaccination with sufficiently high vaccination coverage to break the Rabies transmission cycle. Firstly, there is a clear need to develop field strategies which allow vaccination of large numbers of free roaming dogs in urban areas. Secondly, there is a need for improved methodologies which can rapidly assess whether the vaccination coverage achieved is high enough to result in widespread protective immunity within the dog population. 
This study describes the development and implementation of a mass vaccination programme in Kotagiri, India using mapping techniques and smart phone technology

\section{MATERIALS AND METHODS}

\section{Study area}

Kotagiri $\left(11.43^{\circ} \mathrm{N}, 76.88^{\circ} \mathrm{E}\right)$ is a panchayat town in the Nilgiris district in the Indian state of Tamil Nadu (Fig. 1).


Fig. 1: Google map showing study area (left) and mobile application connected to server (right)

\section{Study design}

This study has two components: Dog vaccination and Survey. On the first day, the dogs were vaccinated and marked and followed up in the $2^{\text {nd }}$ day. The survey was conducted to record the marked and unmarked dogs.

\section{Study population}

Dogs included in the study were those vaccinated, marked and released (VMR) by roaming vaccination teams and those sterilized as a part of the catch-neuter-vaccinate-return (CNVR) programme which ran in parallel to the rotating vaccination work. The study period was from 5th December 2014 to 16th April 2015.

\section{Mission rabies application}

A bespoke 'Mission Rabies' App was developed which enabled information about each dog vaccinated to be recorded on a smart phone on a real-time basis. This information was then synchronized via internet to a web-based server (Fig. 2). The 
dataset for each dog vaccinated included GPS location, manually entered ward number, action taken (vaccinated, marked and released/vaccinated and released but not marked/previously vaccinated within one year, marked/taken to clinic), sex (male/female), ownership status (presented by owner/free roaming), approximate age ( $<3$ months, $>3$ months), neuter status defined by presence/absence of an ear notch routinely performed at the time of surgery (neutered/not-neutered), body condition score (BCS: emaciated (1), underweight (2), healthy weight (3), obese (4), presence of alopecia (four point score of alopecia affecting a percentage of total body surface area; normal (no hair loss), mild ( $<20 \%$ hair loss), moderate (20-80\% hair loss), severe ( $>80 \%$ hair loss)), other disease (transmissible venereal tumour, wounds, lameness, other). Road boundaries were displayed on the app to enable teams to navigate through the ward and stay within boundaries. Three Samsung Galaxy Core 2 phones were used, one with each vaccination team and one with the surveyor.

\section{Vaccinate-assess-move protocol}

Once restrained dogs were vaccinated intramuscularly or subcutaneously (Nobivac ${ }^{\circledR}$ Rabies, MSD Animal Health), they were marked and released. Non-toxic paint along the top and back of the head was used for marking to allow for identification on postvaccination surveys and prevent repeated vaccination.

\section{Post-vaccination survey protocol}

Following completion of vaccinations, a surveyor travelled around the ward by motorbike navigating using the smart phone map to cover every street within the municipality boundaries. Surveys were conducted in the morning between first light and $11 \mathrm{am}$ and in the late afternoon between $3 \mathrm{pm}$ and dusk. Only free roaming dogs were recorded, therefore dogs tied or confined to private property were not recorded. Each dog sighted was recorded in a 'Survey form' on the 'Mission Rabies' app. The data was then synchronized to the central server via internet as described above. A 'path tracker' function was incorporated into the app to record the path travelled during the survey.

\section{Data analysis}

Data summaries and maps can be viewed in real time on the 'Mission Rabies' app backend. Calculations of vaccination coverage were undertaken in Excel 2013 (Microsoft Inc., Redmond, WA). For more detailed analysis, both vaccination and survey datasets were downloaded from the server as CSV files. Road boundaries were imported into ArcGIS Desktop 10.3 and dog sighting (survey) and vaccination locations were labelled with the road according to GPS location. Data was then exported into Excel 2013 for cleaning and analysis. Maps were prepared for presentation in 
QGIS Desktop 2.6.1 (QGIS development team, Open Source Geospatial Foundation Project).



Fig. 2: Real time data and location data of vaccinated dog

\section{RESULTS}

Out of 248 dogs captured or brought by owner, 210 dogs were vaccinated as some dogs were already vaccinated within 1 year and some owners refused to vaccinate. In survey, 147 dogs were sighted of which 77 were observed marked with paints. This indicated a vaccination coverage of $52.4 \%$ (Fig. 4). The total dog population observed during the vaccination programme and survey was 475. Majority of dogs were found to be free roaming $(58.8 \%)$ within vaccinate, mark and release (VMR) of which $51.4 \%$ were male. The neuter status of free roaming and owned dog are shown in Table $1.23 .80 \%$ of the free roaming VMR population were neutered. The male-biased gender ratio is $1.74: 1$. Adults comprised the majority of population $(87.8 \%)$ while puppy represented $12.9 \%$. Adult and puppy vaccination with location data, vaccination coverage with location data and sterilization percentage of male and female stray dog are shown in Fig. 3, Fig. 4 and Fig. 5 respectively. 

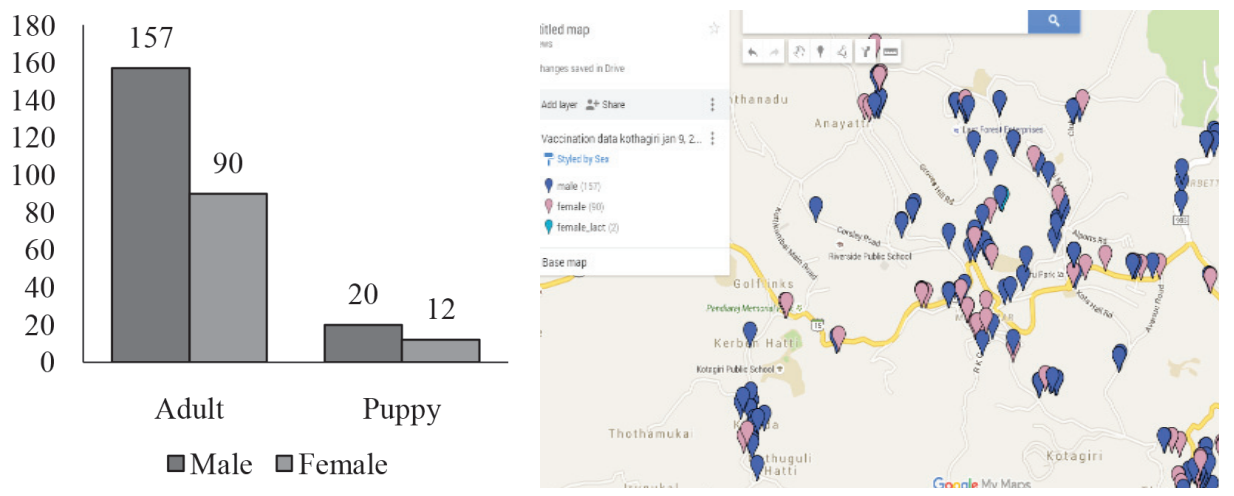

Fig. 3: Adult and puppy vaccination with location data


Fig. 4: Vaccination coverage with location data

Out of 147 dogs sighted, 77 were vaccinated.

Table 1: Neuter status within VMR population for owned and free roaming dog with location data

\begin{tabular}{|l|c|c|c|}
\hline Neuter status & Free Roaming & Presented by owner & Total \\
\hline Entire & 69 & 120 & 189 \\
\hline Neutered & 46 & 13 & 59 \\
Unknown & 1 & - & 1 \\
\hline Total & 115 & 133 & 249 \\
\hline
\end{tabular}




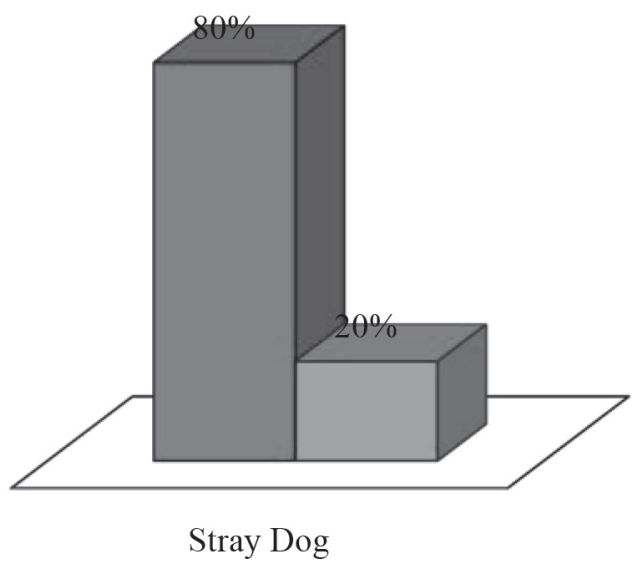

Fig. 5: Sterilization percentage of male and female stray dog DISCUSSION

India has the highest number of human deaths from rabies globally (Hampson, et al., 2015, Knobel et al., 2005). The canine rabies vaccination coverage of more than $70 \%$ dogs has been well documented in numerous countries to significantly reduce the incidence of rabies in both human and canine populations (Jibat et al., 2015, Cleaveland et al., 2003 and Kayali et al., 2003). Despite the pressing need for effective canine rabies vaccination programmes in India owing to highest rabies burden, there have been few publications describing canine rabies vaccination strategies in the country (Kakkar et al., 2012, Shahid et al., 2013 and Davlin et al., 2012). There is a paucity of publications which have demonstrated that a large number of dogs can be vaccinated in a short period of time maintaining a high vaccination coverage.

The vaccination coverage of $52.4 \%$ achieved in this study is higher than the similar study conducted in Indiawho found 34\% (Belsare AV, 2013) and 35.5\% (Reece and Chaaula, 2006) respectively. The geographical area of Kotagiri and dog handling was a challenge in our study. Likewise, misconceptions among owners hindered to achieve higher vaccination coverage (Reece and Chaaula, 2006). In a 12-month period in northwest Tanzania, Hampson et al., 2009, estimated that a target vaccination coverage of $60 \%$ is sufficient to avoid coverage falling below the critical threshold of $40 \%$ between annual campaigns.

Vaccination coverage of $70 \%$ in 16 wards and in 2 wards $68.7 \%$ and $68.8 \%$, the similar study held in Ranchi using Smart Phone Technology (Gibson et al., 2015) which is more than our finding of $52.83 \%$. Smartphone application provided specific functionality of data entry and boundaries displayed on maps, enabled efficient and simple region wise direction of catching and in overall budget for mass vaccination 
campaigns, the cost of using smart phones per dog vaccinated was minimal. Although mobile phone technology has been used in other epidemiological studies (Aanensen, Huntley, Feil and Spratt, 2009), this Ranchi program was the first study to report the development and implementation of a bespoke app tailored towards the collection of data relevant in canine rabies field work. This technology helped the project manager to instantly view maps of where the vaccination teams has been working or download the data in spreadsheet format for estimation of vaccination coverage enabling the prompt direction of teams back to areas with low coverage. This instant access to digitalized data saved significant management time in conversion of paper records into an electronic spreadsheet which made the application of the system more appealing and sustainable at the project management level. The resulting dataset also facilitates study of dog demographics and spatial analysis.

In this study, majority of dog werefound to be free roaming $(58.8 \%)$ and were male (51.4\%). This finding is lower than observed by Gibson et al, 2015 (free roaming 65.6\%), Hibi, 2011 (male 56.5\%). The male biased gender ratio is 1.74:1 observed in this study is in line with the finding of (Pal, 2001) who found it to be 1.37:1. Perception of males as better guard dogs than females has played the role and to avoid the nuisance of owning a bitch in estrus or having to deal with unwanted puppies might be the reason for the observed bias (Daniels and Bekoff, 1989, Daniels, 1983). Unlike in Africa, where a large number of dogs are owned and can be vaccinated through static point vaccine approaches (Cleaveland S et al., 2003), the vast majority of dogs in Kotagiri were not identifiably owned. In this study teams used butterfly nets to catch and restrain the large number of dogs which were not amenable to handling. The use of butterfly nets has been previously described in vaccination campaigns in Bali where the majority of dogs were not amenable to handling (Morters et al., 2014) and has been found to be more effective and humane than other capture methods.

\section{CONCLUSION}

The simple method of marking vaccinated dogs followed by dog-sight surveys to estimate vaccination coverage in the abundant free roaming dog populations is a cheap and effective system to estimate vaccination coverage in real-time. Mass vaccination campaigns provide an ideal opportunity for gathering information about a large cross section of the population with minimal additional effort which can then be used to better direct resources and refine effective methods. Crucially, we developed a 'Mission Rabies' smartphone application (App) which allowed for rapid entry of field data and facilitated the real time assessment of vaccination coverage. This mobile technology ensured that areas of vaccination coverage below $70 \%$ were immediately detected, thereby enabling areas with suboptimal vaccination coverage to be revisited by vaccination teams. If the same approach is rolled out 
more extensively across rabies endemic areas including Nepal, this vaccination strategy has the potential to significantly reduce the incidence of rabies in both dog and human populations. Killing dogs does not stop the disease. Additionally, for developing countries including India and Nepal, open source software like ArcGIS for mapping and epitools for collection of data relevant in canine rabies field can be applied.

\section{REFERENCES}

Aanensen DM, Huntley DM, Feil EJ, al-Own F, Spratt BG. EpiCollect: linking smartphones to web applications for epidemiology, ecology and community data collection. PLoS One. 2009; 4:e6968. doi:10.1371/journal.pone.0006968.

Abbas SS, Kakkar M, Rogawski ET. Costs Analysis of a Population Level Rabies Control Programme in Tamil Nadu, India. PLoS Negl Trop Dis. 2014; 8. doi:10.1371/journal.pntd.0002721.

ANDERSON, R.M., JACKSON, H. C. M., MAY, R. M. \& SMITH, A. M. (1981) Population dynamics of fox rabies in Europe. Nature 289: 765-771

ANON (1988) Report of WHO consultation on dog ecology studies related to rabies control, WHO/Rab Res/88.25. Geneva, WHO.

ANON (2004) Assessing the burden of rabies in India. WHO sponsored national multicentric rabies survey 2003. Bangalore, Association for Prevention and Control of Rabies in India

Anon: WHO expert consultation on rabies: first technical report. In Technical Report Series 931 Geneva, WHO; 2004.

Belsare AV, Gompper ME. Assessing demographic and epidemiologic parameters of rural dog populations in India during mass vaccination campaigns. Prev Vet Med. 2013; 111:139-46. doi:10.1016/j.prevetmed.2013.04.003. Elsevier B.V.

Beran, G. W. (1991) Urban rabies. In the Natural History of Rabies. 2nd edn. Ed G. M. Baer. Boca Raton, CRC Press. pp 427-443

Cleaveland S, Kaare M, Tiringa P, Mlengeya T, Barrat J. A dog rabies vaccination campaign in rural Africa: impact on the incidence of dog rabies and human dog-bite injuries. Vaccine. 2003; 21:1965-73. doi:10.1016/S0264-410X (02)00778-8.

Coleman PG, Fevre EM, Cleaveland S. Estimating the public health impact of rabies. Emerging infectious diseases. 2004; 10(1):140-2. PMID: 15078611 
Coleman PG. \& DYE, C. (1996) Immunisation coverage required to prevent outbreaks of dog rabies. Vaccine 14: 185-186

Conan A, Kent A, Koman K, Konink S, Knobel D. Evaluation of methods for shortterm marking of domestic dogs for rabies control. Prev Vet Med. 2015; 121(12):179-82.

Davlin SL, VonVille HM. Canine rabies vaccination and domestic dog population characteristics in the developing world: A systematic review. Vaccine. 2012; 30:3492-502. doi:10.1016/j.vaccine.2012.03.069.

Fooks A.R., et al., Current status of rabies and prospects for elimination. Lancet, 2014. 384(9951): p. 1389-99. doi: 10.1016/S0140-6736(13)62707-5 PMID: 24828901.

Gangaboraiah, 2007. Assessing the burden of human rabies in India: results of a national multi-center epidemiological survey. Int. J. Infect. Dis. 11, 29-35.

Gibson et al., BMC Infectious Diseases (2015) 15 : 589 DOI 10.1186/s12879-0151320-2.

Gsell AS, Knobel DL, Cleaveland S, Kazwala RR, Vounatsou P, Zinsstag J.Domestic dog demographic structure and dynamics relevant to rabies control planning in urban areas in Africa : the case of Iringa, Tanzania. BMC Vet Res. 2012; 8:236.

Hampson K, Coudeville L, Lembo T, Sambo M, Kieffer A, Attlan M, et al., Estimating the Global Burden of Endemic Canine Rabies. PLoS Negl Trop Dis. 2015; 9.doi:10.1371/journal.pntd.0003709.

Hampson K, Dushoff J, Cleaveland S, Haydon DT, Kaare M, Packer C, et al., Transmission dynamics and prospects for the elimination of canine rabies. PLoS Biol. 2009; 7:e53. doi:10.1371/journal.pbio.1000053.

Hampson K, Dushoff J, Cleaveland S, Haydon DT, Kaare M, Packer C, et al., Transmission Dynamics and Prospects for the Elimination of Canine Rabies. Plos Biology. 2009; 7(3):e1000053. doi: 10.1371/ journal. pbio.1000053 PMID: 19278295.

Hiby LR, Reece JF, Wright R, Jaisinghani R, Singh B, Hiby EF. A mark-resight survey method to estimate the roaming dog population in three cities in Rajasthan, India. BMC Vet Res. 2011; 7:46. doi:10.1186/1746-6148-7-46. BioMed Central Ltd. 
Jibat T, Hogeveen H, Mourits MCM. Review on Dog Rabies Vaccination Coverage in Africa: A Question of Dog Accessibility or Cost Recovery? PLoS Negl Trop Dis. 2015; 9:e0003447. doi:10.1371/journal.pntd.0003447.

Kaare M, Lembo T, Hampson K, Ernest E, Estes A, Mentzel C, et al., Rabies control in rural Africa: evaluating strategies for effective domestic dog vaccination. Vaccine. 2009; 27:152-60. doi:10.1016/j.vaccine.2008.09.054.

Kakkar M, Venkataramanan V, Krishnan S, Chauhan RS, Abbas SS. Moving from rabies research to rabies control: lessons from India. PLoS Negl Trop Dis. 2012;6:e1748. doi:10.1371/journal.pntd.0001748.

Kayali U, Mindekem R, Yémadji N, Vounatsou P, Kaninga Y, Ndoutamia $\mathrm{AG}$, et al., Coverage of pilot parenteral vaccination campaign against canine rabies in N'Djaména, Chad. Bull World Health Organ. 2003; 81:739-44. Available: http://www.pubmedcentral.nih.gov/articlerender. fcgi artid $=2572337 \&$ tool $=$ pmcentrez\&rendertype $=$ abstract .

Knobel DL, Cleaveland S, Coleman PG, Fèvre EM, Meltzer MI, Miranda MEG, et al., Re-evaluating the burden of rabies in Africa and Asia. Bull World Health Organ. 2005; 83:360-8.

Korns RF, Zeissig A. Dog, fox and cattle rabies in New York State: Evaluation of vaccination in dogs. Am J Public Health Nations Health. 1948; 38(1):50-65. PMID: 18917504.

Lankester F, Hampson K, Lembo T, Palmer G, Taylor L, Cleaveland S. Implementing Pasteur's vision for rabies elimination. Science (80-) 2014; 345:1562-4. doi: 10.1126/science.1256306 PMID: 25258065.

Largi, O.P., Arrosi, J.C., Nakajataa. J. \& Villa-Nova, A. (1988) Control of urban rabies. In Rabies. Eds J. B. Campbell, K. M. Charlton. Boston, Kluwer Academic Publishers. pp 407-422.

Lembo T, Hampson K, Kaare MT, Ernest E, Knobel D, Kazwala RR, et al., The feasibility of canine rabies elimination in Africa: dispelling doubts with data. PLoS Negl Trop Dis. 2010; 4:e626. doi:10.1371/journal.pntd.0000626.

Morters MK, McKinley TJ, Horton DL, Cleaveland S, Schoeman JP, Restif O, et al., Achieving Population-Level Immunity to Rabies in Free-Roaming Dogs in Africa and Asia. PLoS Negl Trop Dis. 2014; 8:e3160. doi:10.1371/ journal.pntd.0003160.

Pal SK. Population ecology of free-ranging urban dogs in West Bengal, India. Acta 
Theriol (Warsz). 2001; 46:69-78. doi:10.1007/BF03192418.

Reece JF, Chawla SK. Control of rabies in Jaipur, India, by the sterilisation and vaccination of neighbor-hood dogs. Vet Rec. 2006; 159:379-83.doi:10.1136/ vr.159.12.379.

Shahid S, Kakkar M. Rabies control in India: a need to close the gap between research and policy. Bull World Heal Organ. 2015; 93(2):131-2.

Sudarshan, M.K., Madhusudana, S.N., Mahendra, B.J., Rao, N.S.N., Ashwath Nurayana, D.H., Abdul Raman, S., Meslin, F.X., Lobo, D., Ravikumar, K.,

Suraweera W, Morris SK, Kumar R, Warrell DA, Warrell MJ, Jha P, et al., Deaths from Symptomatically Identifiable Furious Rabies in India: A Nationally Representative Mortality Survey. PLoS Negl Trop Dis. 2012; 6(10):e1847. Doi 10.1371/journal.pntd.0001847 PMID: 23056661.

Totton SC, Wandeler AI, Zinsstag J, Bauch CT, Ribble CS, Rosatte RC, et al., Stray dog population demographics in Jodhpur, India following a population control/ rabies vaccination program. Prev Vet Med. 2010; 97:517.doi:10.1016/j. prevetmed. 2010.07.009. Elsevier B.V.

Vigilato MAN, Cosivi O, Knöbl T, Clavijo A, Silva HMT. Rabies update for Latin America and the Caribbean. Emerg Infect Dis. 2013; 19:678-9. doi:10.3201/ eid1904.121482.

Vigilato MAN, Cosivi O, Knöbl T, Clavijo A, Silva HMT. Rabies update for Latin America and the Caribbean. Emerg Infect Dis. 2013; 19:678-9. doi:10.3201/ eid1904.121482.

Wells C. The control of rabies in Malaya through compulsory mass vaccination of dogs. Bull World Health Organ. 1954; 10:731-42.

WHO. Expert Consultation on Rabies. Second report. 2013. 